\title{
Cancer stem cell differentiation: TGFß1 and versican may trigger molecules for the organization of tumor spheroids
}

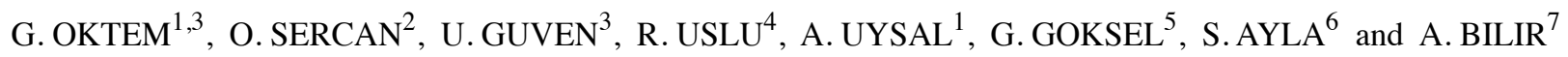 \\ ${ }^{1}$ Department of Histology and Embryology, Faculty of Medicine, Ege University, Bornova 35100, Izmir; \\ ${ }^{2}$ Department of Medical Biology, Faculty of Medicine, Dokuz Eylul University, Narlidere 35340, Izmir; \\ ${ }^{3}$ Department of Stem Cell, Ege University Institute of Health Science, Bornova 35100, Izmir; ${ }^{4}$ Department of Medical \\ Oncology, Faculty of Medicine, Ege University, Bornova 35100, Izmir; ${ }^{5}$ Department of Medical Oncology, \\ Faculty of Medicine, Celal Bayar University, Manisa 45030; ${ }^{6}$ Department of Obstetrics and Gynecology, \\ Zeynep Kamil Gynecology and Maternity Training and Research Hospital, Istanbul 34668; ${ }^{7}$ Department of Histology \\ and Embryology, Emine-Bahaeddin Nakiboglu Medical Faculty, Zirve University, Gaziantep 27100, Turkey
}

Received January 6, 2014; Accepted February 26, 2014

DOI: 10.3892/or.2014.3252

\begin{abstract}
Cancer stem cells (CSCs) have the ability to selfrenew similar to normal stem cells. This process is linked with metastasis and resistance to chemotherapy and radiotherapy. In the present study, we constructed an in vitro differentiation model for CSCs. CSCs isolated and proliferated for one passage were maintained as monolayers or spheroid-forming cells with serum included media for differentiation process. Differentiation of adhesion molecules and cellular ultrastructural properties were investigated and compared in both monolayer and spheroid cultures. $\mathrm{CD} 133^{+} / \mathrm{CD} 44^{+}$cancer-initiating cells were isolated from DU-145 human prostate cancer cell line monolayer cultures and propagated as tumor spheroids and compared with the remaining heterogeneous cancer cell bulk population. Microarray-based gene expression analysis was applied to determine genes with differential expression and protein expression levels of candidates were analyzed by immunohistochemistry. Electron microscopy showed detailed analysis of morphology. TGF $\beta 1$ was found to be significantly upregulated in monolayer CSCs. High expression levels of VCAN, COL7A1, ITG 33 , MMP16, RPL13A, COL4A2 and TIMP1 and low expression levels of THBS1, MMP1 and MMP14 were detected when CSCs were maintained as serum-grown prostate CSC spheroids. Immunohistochemistry supported increased immunoreactivity of TGF $\beta 1$ in monolayer cultures and VCAN in spheroids. CSCs were found to possess multipotential differentiation capabilities through upregulation and/or downregulation of their markers. TGF $\beta 1$
\end{abstract}

Correspondence to: Professor Gulperi Oktem, Department of Histology and Embryology, Faculty of Medicine, Ege University, 11 Ankara Street, Bornova 35100, Izmir, Turkey

E-mail: gulperi.oktem@ege.edu.tr

Key words: cancer stem cells, adhesion molecules, versican, TGF $\beta 1$, spheroid is a triggering molecule, it stimulates versican, Col7A1, ITG $\beta 3$ and, most importantly, the upregulation of versican was only detected in CSCs. Our data support a model where CSCs must be engaged by one or more signaling cascades to differentiate and initiate tumor formation. This mechanism occurs with intracellular and extracellular signals and it is possible that CSCc themselves may be a source for extracellular signaling. These molecules functioning in tumor progression and differentiation may help develop targeted therapy.

\section{Introduction}

Tumors harbor different cell types consisting of complex, phenotypically/genotypically heterogeneous cell populations. Within the tumor mass there resides a rare group of cancer cells known as cancer-initiating or cancer stem cells (CSCs) (1,2). Similar to normal stem cells, CSCs undergo asymmetrical cell division, giving rise to one daughter cell that becomes a committed progenitor. As a result, hierarchies of actively proliferating as well as progressively differentiating cancer cells are formed, contributing to the cellular heterogeneity of human cancers (3). These cells, which are able to self-renew and differentiate, are not only the potential origin of the tumor, but also the possible source of recurrence and chemoresistance (4). Recent studies support this proposal and suggest the utility of several factors to induce the differentiation of CSCs (5).

It has been demonstrated that cells at metastatic sites possess differentiation properties unique to CSCs and have heterogeneous histological features suggesting ability to selfrenew (6). Established cancer cell lines contain CSCs, which can be propagated in vitro using defined conditions to form $3 \mathrm{D}$ tumor spheroids (7). The in vivo cellular microenvironment plays a critical role in the growth and development of both normal and cancer tissues. It is regulated by a complex interplay of soluble factors and signaling molecules secreted by cells. Accumulating data suggest that in vitro three dimensional tumor cell cultures reflect the complex in vitro 
microenvironment more accurately than simple two-dimensional monolayers, especially with respect to gene expression profiles, signaling pathway activity and drug sensitivity $(8,9)$. Also, CSCs spontaneously tend to exist in spheroid formation, as seen in the embroid body formation during development (10). In light of these reports, it may be assumed that spheroids grown in serum contained medium in vitro, may reflect the differentiation properties of CSCs better when compared to other in vitro models. Spheroids have been used as a metastasis model in several studies (11-13).

We hypothesized that the differentiation process in CSCs, when compared to non-CSCs, could be followed by the adhesion molecules that are expressed on the cells. These molecules could provide the means for the future establishment of therapeutic strategies in cancer. In the present study, we described approaches to image and analyze the differentiation properties of human prostate CSCs within 3D spheroids. We believe our approach may serve as a model for defining expression and protein profiles of CSCs in a 3D environment.

\section{Materials and methods}

Cell culture conditions and reagents. The DU145 human prostate cancer cell line was supplied by the American Type Culture Collection (ATCC; Rockville, MD, USA) and was grown in monolayer culture in Dulbecco's modified Eagle's medium-F12 (DMEM-F12; Biological Industries, Israel) supplemented with $10 \%$ heat-inactivated fetal calf serum, $100 \mathrm{U} / \mathrm{ml}$ penicillin and $100 \mu \mathrm{g} / \mathrm{ml}$ streptomycin (Sigma Chemical Co., St. Louis, MO, USA). Cells in semi-confluent flasks were harvested using $0.05 \%$ trypsin (Sigma Chemical Co.), centrifuged after addition of DMEM-F12 for trypsin inactivation, and then resuspended in culture medium. Antibodies used for immunohistochemistry and western blot analysis were TGF $\beta 1$, versican, CDH1 (Abcam), ICAM1, Col7A1, ITGß3, MMP1, MMP14, MMP16 (Bioss), NCAM1 (Abcam), SPP1 (Bioss), THBS1 (Abcam).

Fluorescence activated cell sorting and experimental groups. For fluorescence activated cell sorting (FACS), cells were detached using non-enzymatic cell dissociation solution (Sigma). Approximately 56,100 cells were incubated with antibody, diluted 1:100 in FACS wash $(0.5 \%$ bovine serum albumin; $2 \mathrm{mM} \mathrm{NaN}_{3} ; 5 \mathrm{mM}$ EDTA), for $15 \mathrm{~min}$ at $4^{\circ} \mathrm{C}$. For controls, an isotype and concentration matched $\mathrm{PE}$ labeled control antibody was used as well as samples labeled with PE attached CD133/1 (clone AC133/1) (both from Miltenyi Biotec, UK) and FITC labeled CD44 (clone G44-26, BD Pharmingen). After three 5 min washes (with FACS wash), the cells were re-suspended and sorted for a CD133 $3^{\text {high }} / \mathrm{CD} 44^{\text {high }}$ population. Both the sorted cell population and its remaining non-sorted counterpart were collected for further analyses. The two separate cell populations were cultured in two different settings; either as monolayer 2D culture or as 3D multicellular tumor spheroids.

Construction of spheroids and sphere formation assay. The clonogenic potential of phenotypically different populations was analyzed in 3D non-adherent cell culture conditions. Tumor cells grown as a monolayer were re-suspended, with trypsin, counted, seeded $10^{3}$ cells/well and cultured over $3 \%$ Noble agar-coated 6-well culture plates (Difco, USA). Two weeks after initiation, plates were inspected for colony (sphere) growth. The number of colonies within each well was counted under the microscope and representative fields were photographed. First passage floating spheres were removed, gently disaggregated and transferred to a new $3 \%$ Noble agarcoated well.

PCR array assay. Total RNA was extracted from sorted cells and non-sorted counterparts (miRNeasy kit; Qiagen, Germany). Synthesis of cDNA was carried out using C-03 RT2 First Strand kit (SA Biosciences, Frederick, MD, USA). Stem cell-specific gene expression profiles were analyzed by PCR Array Assay (Custom Panel 384; Roche) in accordance with the manufacturer's recommendations. Briefly, total RNA was isolated from monolayer cell populations or whole floating spheroids. Up to $1 \mu \mathrm{g}$ of total RNA was treated with DNase and cDNA was prepared using RT2 First Strand kit. Pairs of the test and control cDNA samples were added to RT2 qPCR master mix and distributed across the 96-well PCR array plates. Each plate contained 84 adhesion molecule-related probes and control housekeeping genes. Triplicate assays were performed. Following real-time PCR [LightCycler 480 (LC 480); Roche Molecular Systems], amplification data (foldchanges in $\mathrm{Ct}$ values of all the genes) was analyzed by software and $\geq 1.5$ fold-change was used as filtering criteria. The list of genes analyzed for differential expression is as follows: ADAMTS1 (ADAM metallopeptidase with thrombospondin type 1 motif, 1); COL11A1 (collagen type XI, $\alpha 1$ ); COL4A2 (collagen type IV, $\alpha 2)$; VCAN (versican); ECM1 (extracellular matrix protein 1); ITGA3 (integrin $\alpha 3$ ); ITGAL (integrin $\alpha \mathrm{L}$ ); ITG $\beta 4$ (integrin $\beta 4$ ); LAMB1 (laminin $\beta 1$ ); MMP11 (matrix metallopeptidase 11); MMP16 (matrix metallopeptidase 16); $M M P 9$ (matrix metallopeptidase 9); SELP (selectin P); $T G F \beta 1$ (transforming growth factor $\beta 1$ ); TIMP2 (TIMP metallopeptidase inhibitor 2); VTN (Vitronectin); ADAMTS13 (ADAM metallopeptidase with thrombospondin type 13 motif, 1); COL12A1 (collagen $12 \alpha 1$ ); COL5Al (collagen 12 $\alpha 1)$; $C T G F$ (connective tissue growth factor); FN1 (fibronectin 1); ITGA4 (integrin $\alpha 4$ ); ITGAM (integrin $\alpha \mathrm{M}$ ); ITG $\beta 5$ (integrin $\beta 5$ ); LAMB3 (laminin $\beta 3$ ); MMP12 (matrix metallopeptidase 12); MMP2 (matrix metallopeptidase 2); NCAM1 (neural cell adhesion molecule 1); SGCE (sarcoglycan- $\varepsilon$ ); THBS1 (thrombospondin-1); TIMP3 (TIMP metallopeptidase inhibitor 3); GAPDH (glyceraldehyde-3-phosphate dehydrogenase); ADAMTS8 (ADAM metallopeptidase with thrombospondin type 1 motif, 8); COL14A1 (collagen XIV, a1); COL6A1 (collagen VI, $\alpha 1$ ); CTNNA1 (catenin $\alpha 1$ ); HAS1 (hyaluronan synthase 1); ITGA5 (integrin $\alpha 5$ ); ITGAV (integrin $\alpha \mathrm{V}$ ); KAL1 (Kallmann syndrome 1); LAMC1 (laminin $\gamma 1$ ); MMP13 (matrix metallopeptidase 13); MMP3 (matrix metallopeptidase 3); PECAM1 (platelet endothelial cell adhesion molecule 1); SPARC (secreted protein acidic and rich in cysteine); THBS2 (thrombospondin-2); CLEC3B (C-type lectin domain family 3 , member B); $A C T B$ ( $\beta$-actin); $C D 44$ (cell surface glycoprotein CD44); COL15A1 (collagen XV $\alpha 1)$; COL6A1 (collagen VI $\alpha 1)$; CTNNB1 ( $\beta$ catenin); ICAMI (intercellular adhesion molecule 1); ITGA6 (integrin $\alpha 6$ ); ITG $\beta 1$ (integrin $\beta 1$ ); LAMA1 (laminin, $\alpha 1$ ); MMP1 (matrix 

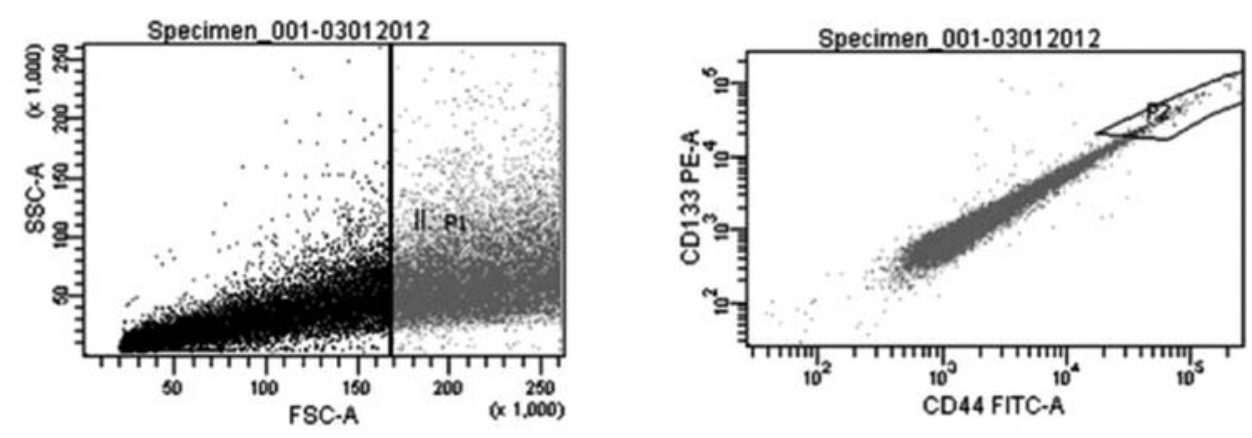

Figure 1. Prostate cancer stem cells sorted with FACSAria. CD133 high $/ C D 44^{\text {high }}$ populations presented in P2. Aside from this population, the remaining cells were classified as non-sorted cells.

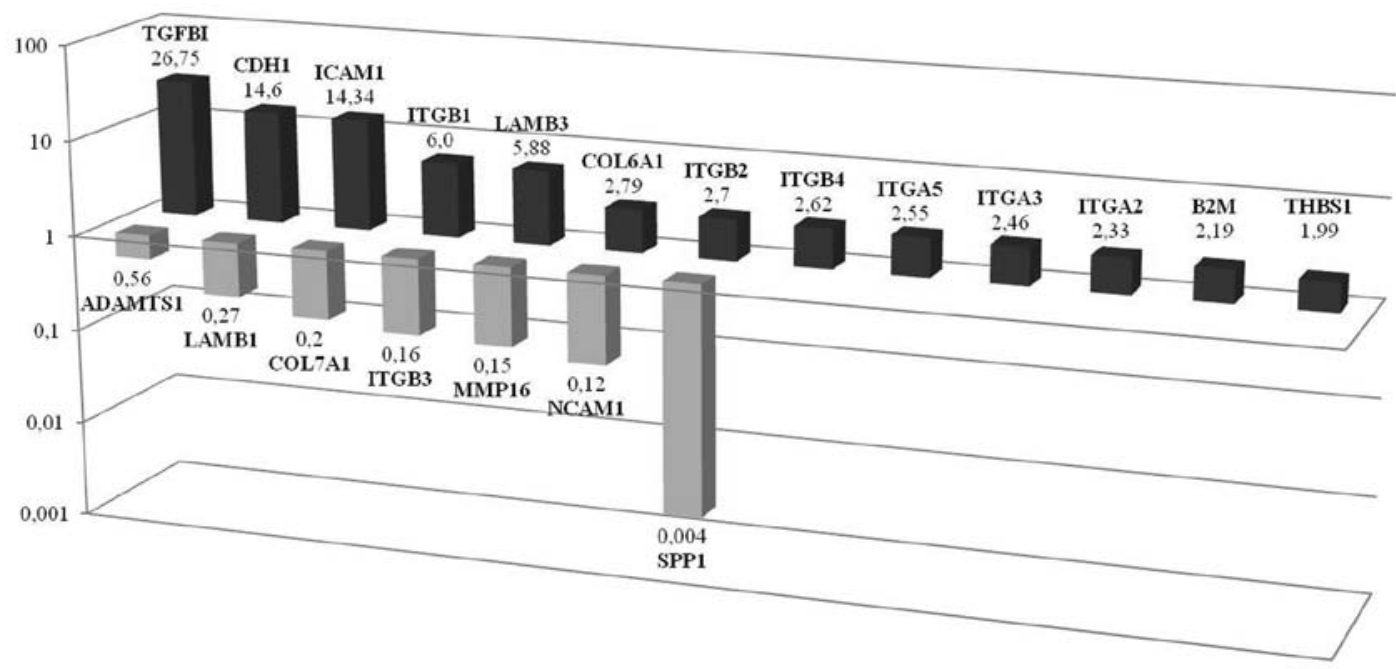

Figure 2. TGF $\beta 1, C D H 1$ and ICAM1 gene expressions are significantly upregulated in monolayer $\mathrm{CD} 133^{+} / \mathrm{CD} 44^{+} \mathrm{CSC}$ s according to non-CSCs. SPP1 was the most downregulated gene in this group.

metallopeptidase 1); MMP14 (matrix metallopeptidase 14); MMP7 (matrix metallopeptidase 7); SELE (E-selectin); SPG7 (spastic paraplegia 7); THBS3 (thrombospondin 3); TNC (tenascin C); RPL13A (ribosomal protein L13a); CDH1 (cadherin 1, type 1); COL16A1 (collagen XVI $\alpha 1$ ); COL7AI (collagen VII $\alpha 1$ ); CTNND1 (catenin $\delta 1) ; I T G A 1$ (integrin $\alpha 1$ ); ITGA7 (integrin $\alpha 7$ ); ITG $\beta 32$ (integrin $\beta 2$ ); LAMA2 (laminin, $\alpha 2)$; MMP10 (matrix metallopeptidase 10); MMP15 (matrix metallopeptidase 15); MMP8 (matrix metallopeptidase 8); SELL (selectin L); SPPI (secreted phosphoprotein 1); TIMP1 (TIMP metallopeptidase inhibitor 1); VCAM1 (vascular cell adhesion molecule 1$) ; B 2 M$ ( $\beta$-2-microglobulin); CNTN1 (contactin 1); COLIAl (collagen I $\alpha 1$ ); COL8A1 (collagen VIII $\alpha 1) ; C T N N D 2$ (catenin $\delta 2$ ); ITGA2 (integrin $\alpha 2$ ); ITGA 8 (integrin $\alpha 8$ ); ITG $\beta 3$ (integrin $\beta 3$ ); LAMA3 (laminin, $\alpha 3$ ); HPRT1 (hypoxanthine phosphoribosyltransferase 1).

Western blot analysis. Cell pellets were lysed in Mammalian Protein Extraction Reagent (M-PER; Thermo Fisher Scientific, Rockford, IL, USA). Following centrifugation at 14,000 x g for $15 \mathrm{~min}$, protein concentrations were quantified (in duplicate) by the Bradford method (Bio-Rad Laboratories, Hercules, CA, USA). Equal amounts of protein were run on SDS polyacrylamide gel electrophoresis (PAGE) and transferred to nitrocellulose membranes (Bio-Rad). Membranes were blocked with $5 \%$ non-fat dry milk prepared in Tris-buffered saline containing $0.1 \%$ Tween-20 (TBST) at room temperature for $1 \mathrm{~h}$. The membrane was then incubated with primary antibodies at $4^{\circ} \mathrm{C}$ overnight. Antibodies (Cdh1 and Thbs1) were obtained from Abcam (Abcam Ltd., UK) and prepared according to the manufacturer's instructions. Following several washes in TBST, membranes were incubated with appropriate secondary antibodies (1:100 dilutions; Millipore Upstate USA, Charlottesville, VA, USA) at room temperature for $1 \mathrm{~h}$. Protein bands were visualized by the Kodak Gel Logic 1500 Imaging System.

Immunohistochemical analysis. Our immunohistochemistry protocols were published previously (14). Briefly, monolayer cells were maintained in 24-well plates and fixed with paraformaldehyde; spheroids were processed by routine histological processing and embedded in paraffin wax. Cells were initially incubated with primary antibodies overnight at $40^{\circ} \mathrm{C}$ in a humidity chamber, followed by a modified streptavidin-peroxidase treatment. After incubation with DAB (Invitrogen Ltd., UK), sections were counterstained 
with Mayer's hematoxylin (Sigma Chemical Co., St. Louis, MO, USA). Immunoreactivity of molecules was assessed by light microscopy using an Olympus BX-51 with an Olympus C-5050 digital camera. Staining was graded independently by two investigator-blind specialists. Evaluation was carried out semi-quantitatively on the following scale: mild, moderate and strong.

Transmission electron microscopy. Harvested monolayers and spheroids were fixed with $2.5 \%$ glutaraldehyde in $0.1 \mathrm{M}$ sodium cacodylate buffer and post-fixed in $1 \%$ osmium tetroxide $/ 0.1 \mathrm{M}$ sodium cacodylate buffer for $1 \mathrm{~h}$ at $4^{\circ} \mathrm{C}$. Cells were incubated in $1 \%$ uranyl acetate for $1 \mathrm{~h}$ at $4^{\circ} \mathrm{C}$, dehydrated in graded acetone series, and embedded in Epon 812. Samples were cut using a rotating-blade microtome (Leica, Heerbrugg, Switzerland). Sections $(70 \mathrm{~nm})$ were mounted on copper grids. Sections were subsequently stained with 5\% uranyl acetate and counterstained with Reynold's lead citrate. Sections were examined using a JEOL JEM 1011 transmission electron microscope.

\section{Results}

The purity of $C D 133^{\text {high }} / C D 44^{\text {high }}$ sorted and non-sorted subpopulations and sorting rates. DU145 human prostate cancer cells were separated with FACS as CD133 $3^{\text {high }} / \mathrm{CD} 44^{\text {high }}$ population (sorted cells) and non-sorted counterparts (Fig. 1). In order to assess the degree to which genetic imbalances were observed in CSCs vs. non-CSCs, we compared array profiles of both cell types and the two culture conditions that they were grown in (monolayer and spheroids).

Prior to the PCR expression microarray, purity of CSC and non-CSC samples was tested with CD133 and CD44 antibodies. Sorting rate analysis and purity of cells were evaluated sequentially. Rates were $96,7 \pm 5,4 \%$ for sorted cells and $90,33 \pm 5,4$ for non-sorted cells. In order to confirm the flow cytometry analyses, cells were re-evaluated after sorting and the analyses were repeated after one passage. Results showed the cell purity after sorting was $85 \%$. Immunofluorescence staining yielded a cell purity of $>85 \%$ in all samples.

TGF 1 triggers molecules in monolayer $C D 133^{+} / C D 44^{+}$ prostate cells when cells arrange for $3 D$ composition. TGF $\beta 1$, $C D H 1$ and ICAMI gene expressions were significantly upregulated in $\mathrm{CD} 133^{+} / \mathrm{CD} 44^{+} \mathrm{CSC}$ grown as a monolayer when compared to their CD133/CD44- counterpart. From the latter candidates' gene, we validated the ten top-ranked genes and ITG $\beta 1, L A M B 3, I T B G 2, I T G A 2, C O L 6 A 1, I T B 4$, ITGA3, THBS, B2M and ITGA5 were observed to be markedly upregulated. $T G F \beta 1$ is emphasized since it is the most upregulated gene in monolayer cells. Secreted phosphoprotein 1 (SSP1) is observed to be the most downregulated gene in monolayer CSCs. Additionally NCAM1, MMP16, ITG $\beta 3$, COL7A1, LAMB1, ADAMTS1 were also downregulated (Fig. 2). Immunohistochemistry of monolayer $\mathrm{CD} 133^{+} / \mathrm{CD} 44^{+}$ cells also showed the strong immunoreactive staining of TGF 1 1, CDH1, ICAM1 and THBS1 when compared to the bulk counterpart. In addition, staining intensity was significantly decreased for SPP1, NCAM1, MMP16, ITG $\beta 3$ and COL7A1 (Fig. 3).

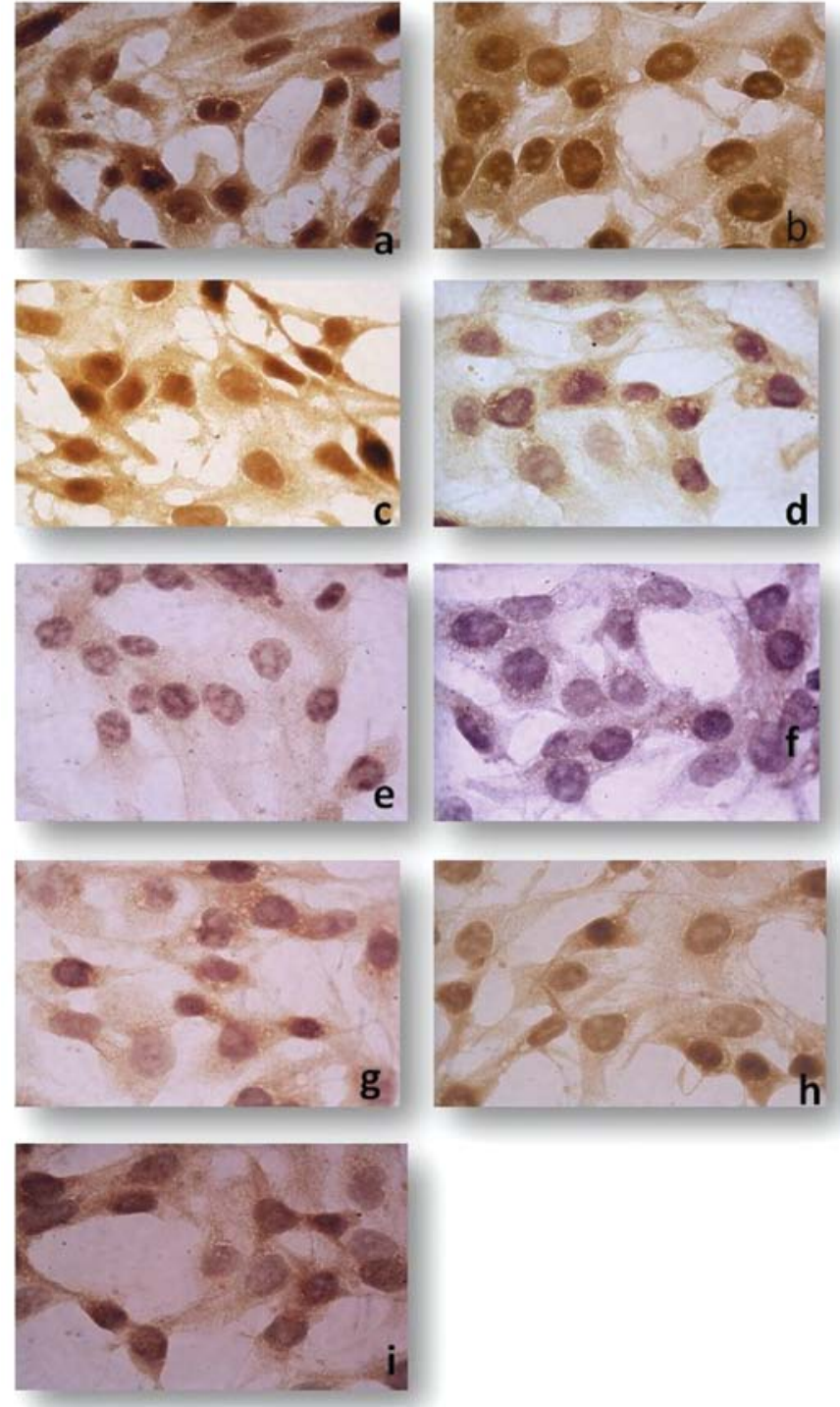

Figure 3. Immunohistochemical analysis of (a) TGF 1 , (b) CDH1, (c) ICAM1, (d) THBS1, (e) SPP1, (f) NCAM1, (g) MMP16, (h) ITG 33 and (i) Col7A1 was demonstrated in monolayer CSCs. TGF $\beta 1$ and $\mathrm{CDH} 1$ immunoreactivity significantly increased in these cells.

ITG 33 upregulation and PECAM1 downregulation are characteristic of CSCs with respect to non-CSCs in spheroids. Significant differences were observed mainly in this group according to adhesion molecules between CSC and non-CSC populations grown as spheroids. Array results showed that $I T G \beta 3, I T G A 2, I T G A 5$ and $S P G 7$ were upregulated, while PECAM1, ADAMTS1, VCAN, CTNND2, TNC, ACTB, COL12A1, MMP14, TGF $\beta 1, S P P 1$ and ITGA6 were downregulated in CSCs. ITG $\beta 3$ was the third upregulated gene in CSC spheroids when compared with the differential expression in monolayer cells. A significant increase was observed in CSC spheroids when we compared with the non-CSC population. It is of note that VCAN was significantly high (46-fold change) in CSC spheroids when compared to its monolayer counterpart. However, VCAN is downregulated in CSC spheroids when compared to its non-CSC spheroid counterpart (Fig. 4).

VCAN expression significantly increases in $\mathrm{CD} 133^{+} / C D 44^{+}$ prostate cancer cells propagated as tumor spheroids. DU145 


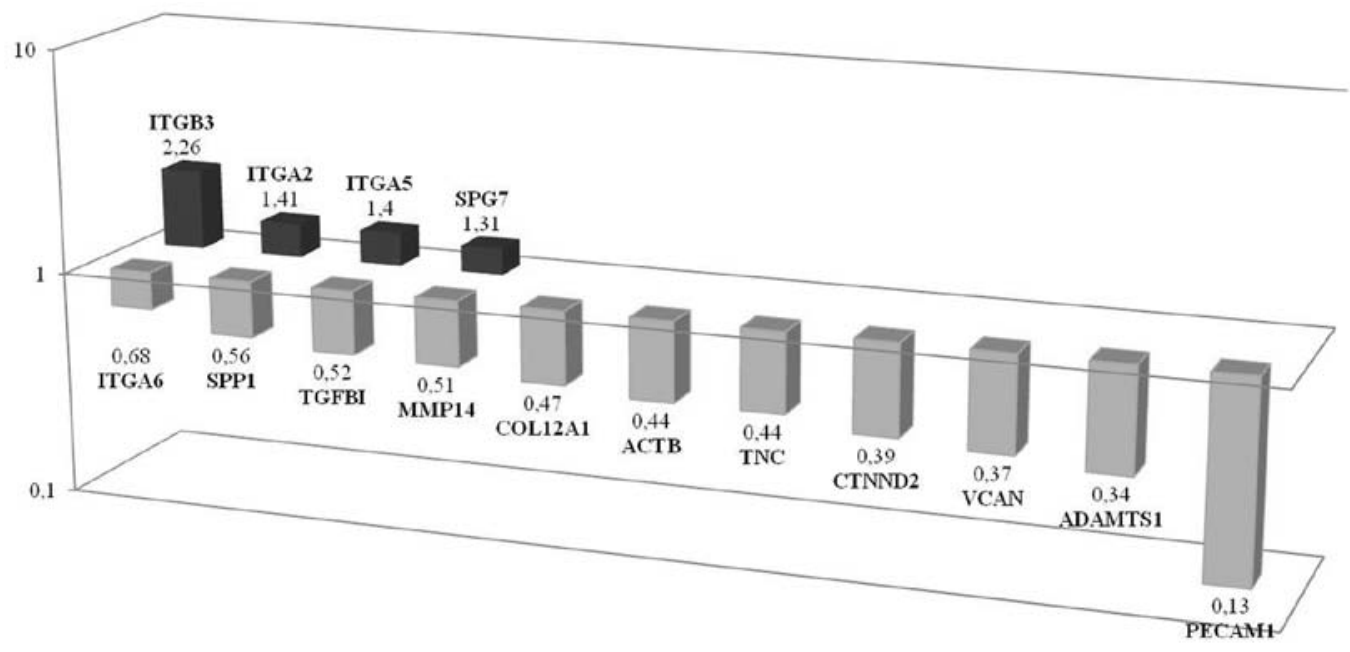

Figure 4. Differences of genetic profile between CSC and non-CSC populations investigated in spheroids in adhesion molecules. Results of array showed that ITG 33, ITGA2, ITGA5 and SPG7 were upregulated while PECAM1, ADAMTS1, VCAN, CTNND2, TNC, ACTB, COL12A1, MMP14, TGF 1 , SPP1 and ITGA6 were downregulated, respectively, in CSCs according to non-CSCs.

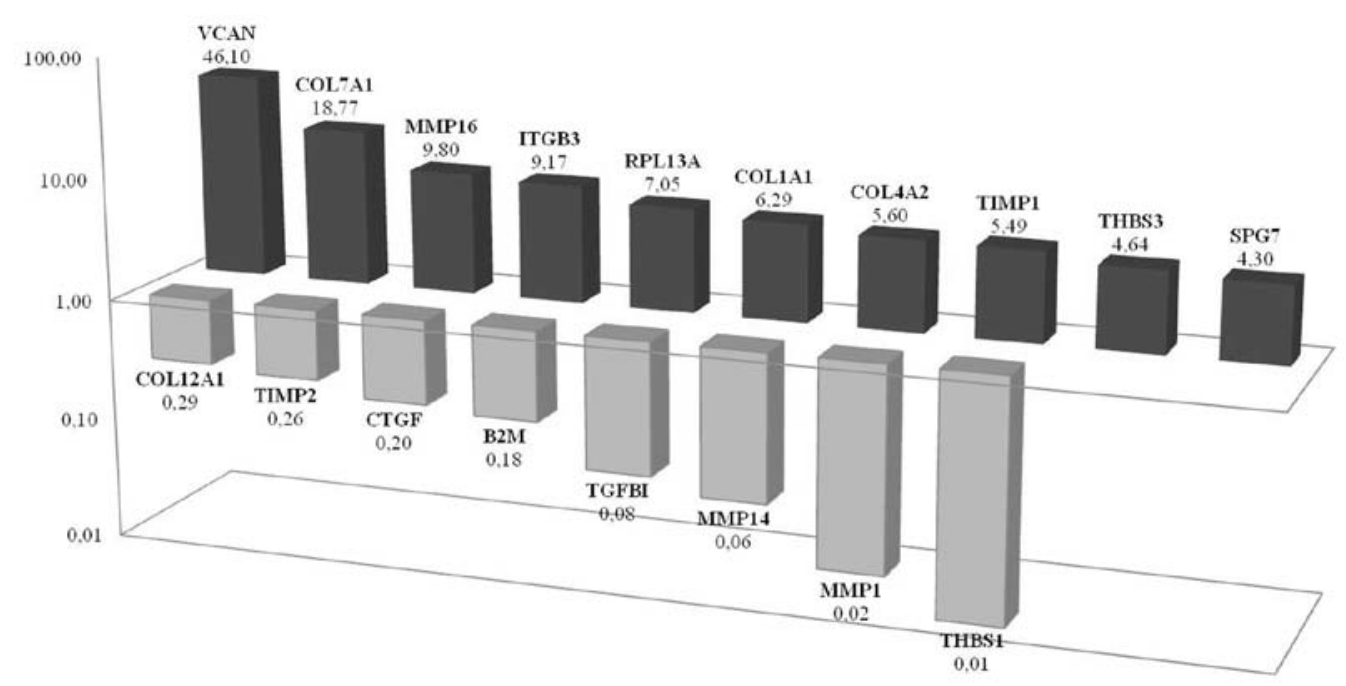

Figure 5. Differentiated CSC-related genes. Spheroid-forming cells originated from CD133 $/ \mathrm{CD} 44^{+}$monolayers showed elevated expression of $V C A N$, COL7A1, ITG $33, M M P 16, R P L 13 A, C O L 4 A 2$ and TIMP1 according to monolayer. The most significant change was demonstrated in VCAN where a 46-fold change was observed.

tumor spheroids were cultured in low-adherence culture conditions as described above and maintained in culture for 10-14 days. Spheroid forming CD133 ${ }^{+} / \mathrm{CD} 44^{+}$cells showed elevated expression of VCAN, COL7A1, ITG $\beta 3, M M P 16$, $R P L 13 A, C O L 4 A 2$ and TIMP1 when compared with the $\mathrm{CD} 133^{+} / \mathrm{CD}_{4} 4^{+}$monolayer cells they originated from. The most significant change was demonstrated in VCAN expression where a 46-fold change was observed (Fig. 5). Increased VCAN expression was also demonstrated by immunohistochemistry in CSC spheroids when compared to other cell groups. On the other hand, THBS1 is the most downregulated gene in CSC spheroids when compared with CSC monolayers. PCR expression array results showed that $M M P 1, M M P 14, T G F \beta 1$, B2M, CTGF, TIMP2 and COL12Al were all downregulated in prostate CSC spheroids. Western blot analysis of Thbs1 showed an increased protein level in monolayer CSCs when compared with spheroid CSCs. However, the most significant decrease for THBS1 was observed in monolayer forming non-CSCs (Fig. 7b). Immunohistochemistry supported the increased expression in CSC spheroids for VCAN, COL7A1 and MMP16 molecules (Fig. 6).

CDH1 expression is significantly increased in non-CSCs propagated as tumor spheroids. PCR-array analyses performed in non-CSCs that were grown in culture as spheroids showed that CDH1, PECAM1, COL4A2, GAPDH, FN1, ITG $\beta 4$, COL1A1, TNC, RPL13A, CTNND2, MMP15, ICAM1, MMP9, ITG $\beta 1$ and $T G F \beta 1$ expressions were significantly upregulated while MMP1, THBS1, SPP1, LAMB1, MMP14, TIMP2, B2M and ITGA6 expressions were downregulated. Among these molecules, the most upregulated gene was observed to be CDH1 and the most downregulated MMPI. It should be noted that in the previous experiments where we compared the spheroid and monolayer CSCs groups, VCAN was the most upregulated 

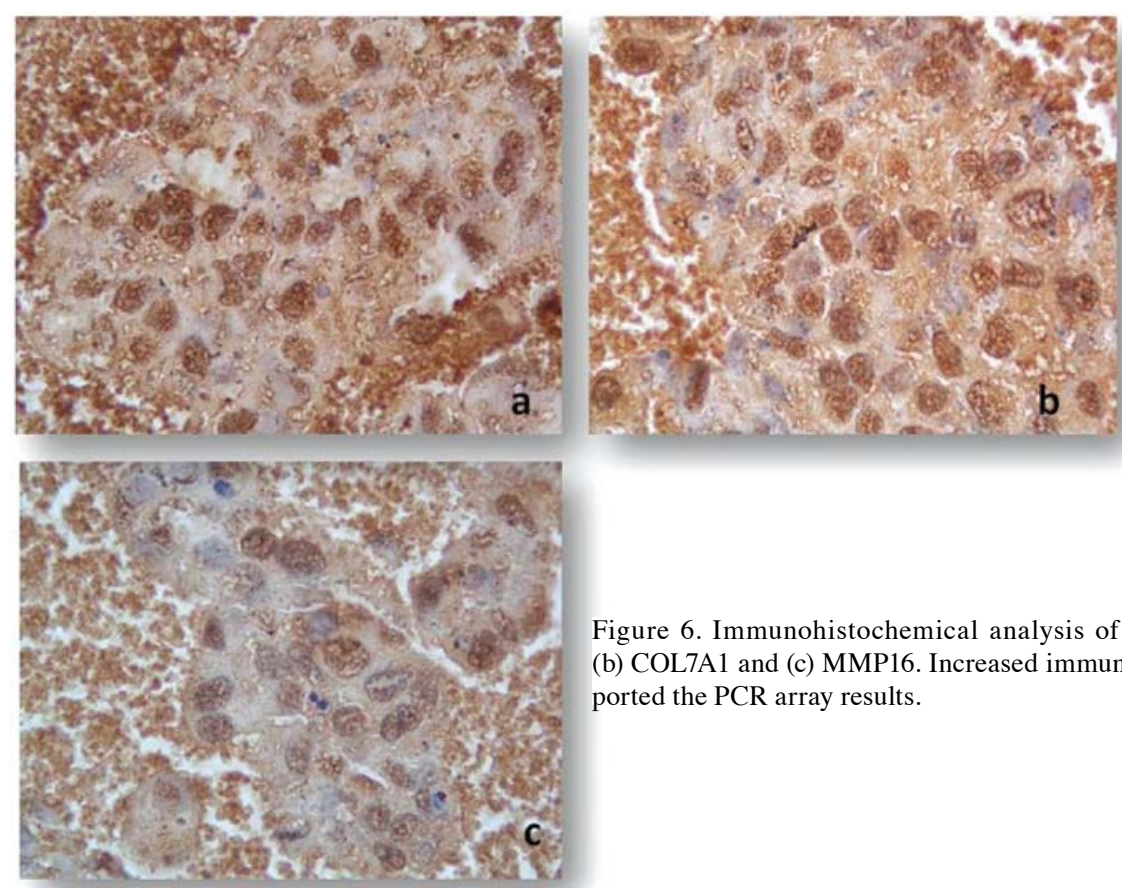

Figure 6. Immunohistochemical analysis of CSC spheroids. (a) VCAN (b) COL7A1 and (c) MMP16. Increased immunohistochemical staining supported the PCR array results.

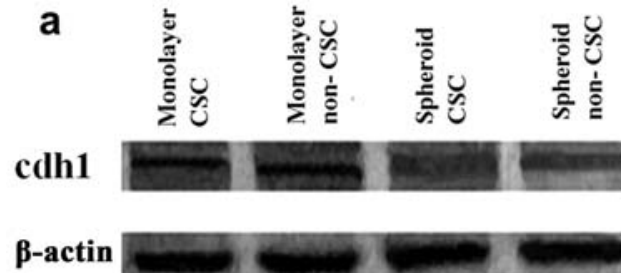

kDa thbs1

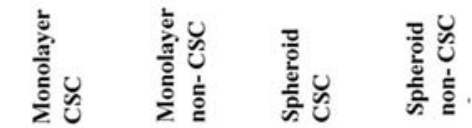

\section{2 kDa $\beta$-actin}

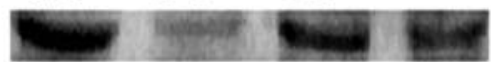

kDa

Figure 7. Western blotting showing (a) the increased CDH1 protein levels in non-CSC spheroids according to monolayer CDH1. (b) Thbs1 showed increased protein level in monolayer CSCs when compared to spheroid CSCs. However, the most significant decrease for THBS1 was observed in monolayer non-CSCs.

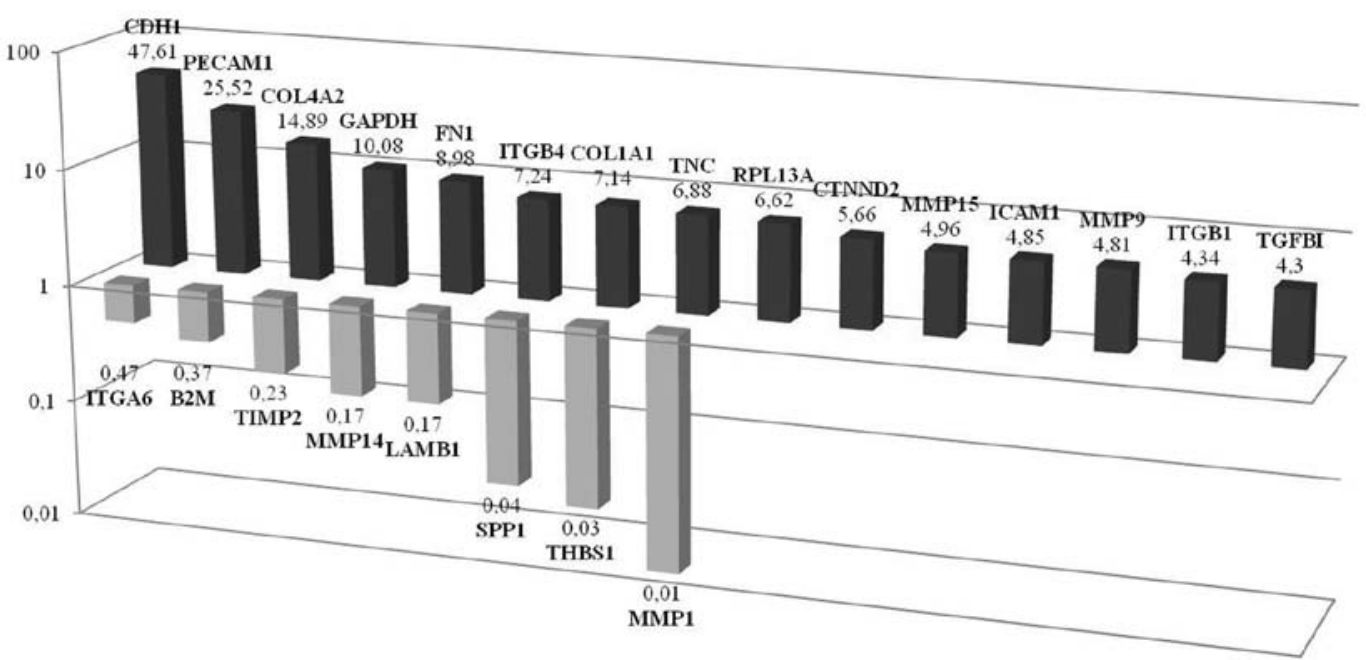

Figure 8. PCR-array analysis performed in the cells which sorted to be non-CSCs and maintained to be spheroids. Results showed that $C D H 1, P E C A M 1$, COL4A2, GAPDH, FN1,ITGB4, COL1A1, TNC, RPL13A, CTNND2, MMP15, ICAM1, MMP9, ITGB1 and TGFß1 were significantly upregulated while MMP1, THBS1, SPP1, LAMB1, MMP14, TIMP2, B2M and ITGA6 were downregulated, respectively.

and THBS1 was the most downregulated gene. At this point, our results suggest that these differences may be an important lead to fully characterize the mechanisms of cell organiza- tion (Fig. 8). Western blotting also showed increased CDH1 protein levels in non-CSC spheroids when compared to its monolayer counterpart (Fig. 7a). 

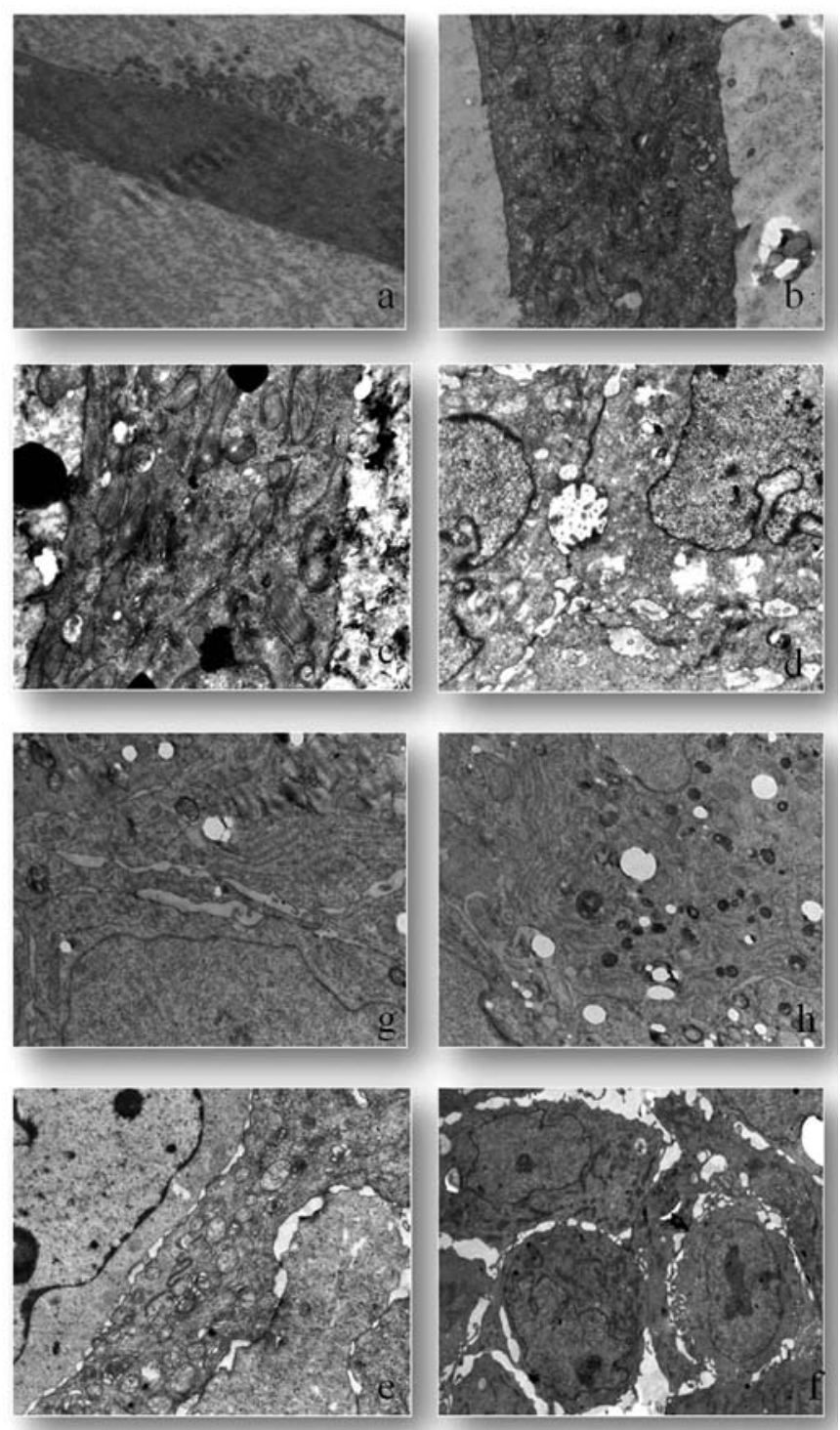

Figure 9. CSCs appear in monolayer cells with intact cell and nuclear membranes. (a) These cells have villous-like protrusions over the surface and these villus-like structures were observed. (b) Non-CSC population showed increased organelles and villus protrusions were not observed (c) Vacuolization and mitophagy increased in non-CSCs. (d) When cells maintained as spheroids distinct from monolayer CSCs villous-like structures, intercellular lacunae were determined. (e) In CSC spheroids, the most increased organelle was the golgi. (f) However, the cellular organelles were observed to be clustered in part of the cytoplasm. (g) Most increased organelle was granular endoplasmic reticulum in non-CSC spheroid. (h) Autophagic vacuoles significantly increased in non-CSCs.

Three dimensional composition changes cell ultrastructure. CSCs grown as a monolayer had intact cell and nuclear membranes. These cells have villous-like protrusions over the surface. These villi-like structures are observed abundantly in nucleus adjacent cell membrane domains (Fig. 9a). Non-CSC populations were observed to have an increased size and number of their organelles such as golgi, mitochondria and granular endoplasmic reticulum. The villous-like structures observed in CSCs were not detected in this group (Fig. 9b). Vacuolization and mitophagy increased in non-CSCs (Fig. 9c). Electron microscopic investigations were performed on cells maintained as spheroids. These tight intercellular connections were observed to be intermittently interrupted along membranes and resulted in cellular gaps. The villous-like protrusions observed in monolayer CSCs were also observed in $\mathrm{CSC}$ spheroids. However, as a distinctive feature, these villouslike structures were observed in intercellular lacunae (Fig. 9d). In CSC spheroids, the organelle most increased in size was the golgi (Fig. 9e). However, the cellular organelles were observed to be clusters in the cytoplasm (Fig. 9f). Increased lipid depositions were observed in cytoplasm and among organelles. Non-CSC spheroids indicated euchromatic nucleus and smooth nuclear membrane. As for spheroid non-CSCs, the most increased organelle was observed to be the granular endoplasmic reticulum (Fig. 9g). Another important observation of the electron microscopic analyses was the significant increase of autophagic vacuoles in non-CSCs (Fig. 9h).

\section{Discussion}

Our data suggest that $\mathrm{CD} 133^{+} / \mathrm{CD}_{4} 4^{+}$prostate cancer stem cells (CSCs) affect their microenvironment and the cellular signaling in surrounding tissue resulting in changes in their behavior reflected as different expression profiles. When CSCs constitute a complex and organized formation, versican is the highest upregulated gene among adhesion molecules. We believe it is important that versican upregulation is only observed in $\mathrm{CD} 133^{+} / \mathrm{CD}_{4} 4^{+}$prostate $\mathrm{CSC}$ s and that this upregulation may be specific for CSCs. Versican is a hyalectan, a specific proteoglycan that affects cell signaling, motility, adhesion, growth and apoptosis. Changes in the differential expression of proteoglycans in a specific tissue may promote or inhibit tumor progression (15). In prostate cancer, versican produced by prostatic fibroblasts inhibited the attachment of the tumor cells to fibronectin. This inhibition provides increased tumor cell motility and facilitates local invasion and possibly metastasis $(16,17)$. CD44 is a hyaluronan receptor and it displays increased expression in the CSC populations of several types of cancer. Formation of the hyaluronan-CD44-versican complex plays an effective role in the assembly of a polarized pericellular sheath to promote tumor cell motility $(18,19)$. We demonstrated increased gene expression in prostate CSC spheroids, which could have resulted from tumor cells increasing the transcription of this molecule during the cellular organization. This observation correlates with other data in our study such as the upregulation of transforming growth factor $\beta 1$ (TGF $\beta 1$ ) in monolayer CSCs. Our study suggests the tumor cells themselves are the source of versican, and it has previously been reported that $T G F \beta 1$ can induce versican production in prostate cancer cells (20). It is possible to assume that during tumor organization, TGF $\beta 1$ is upregulated initially creating an environment favoring the organization of the hyaluronan-CD44-versican complex in prostate CSCs. In addition, decrease in ADAMTS (a disintegrin and metalloproteinase with trombosondin motifs) is found to accompany the enhanced expression of versican by TGF $\beta 1$ (21). Our data reveals a decreased ADAMTS expression in CSC spheroids, where versican accumulation may be a secondary response to ADAMTS degradation. Knaup et al demonstrated that activated TGF $\beta$ signaling (either in response to wounding or to simulate type VII collagen expression) could facilitate cancer development and progression (22). In the present study, we demonstrated significant upregulation 
in COL7A1 expression in CSC spheroids and this is probably related to the positive stimuli of TGF $\beta 1$ signaling. On the other hand, COL7A1 functions as an anchoring fibril between the external epithelia and the underlying stroma. Mutations in this gene are associated with all forms of dystrophic epidermolysis bullosa (23). In the present study, increased COL7A1 in sorted spheroids suggest that proliferated CSCs used this molecule for anchoring to the extracellular matrix.

Integrins are transmembrane adhesion molecules that mediate cell-cell and cell-extracellular matrix attachment (24). Integrins can influence cell migration and invasion by directly mediating adhesion to the extracellular matrix or regulating intracellular signaling pathways that control cytoskeletal organization, force dependent effects and survival (25). These glycoproteins regulate cell growth, proliferation, migration and apoptosis and, as a consequence, can have a potential role in tumor progression and metastasis with the aberrant expression of its genes $(26,27)$. The ex vivo expansion of stem cells using extracellular proteins such as fibronectin and laminin enhances the homing and differentiation of these cells and also promotes the upregulation of $\beta 1$ integrins $(28,29)$. In addition, vitronectin is a main receptor for integrin $\alpha 5 \beta 3$ and is directly related to differentiation of CSCs (6). In the present study, we observed a differential integrin expression in which there was a predominant overexpression in CSC spheroids. ITG $\beta 3$ (CD61) was found to be significantly high in this cell population. Lo et al recently deciphered the link in the integrin $\beta 3$-TGF $\beta$ signaling mode and the mechanisms underlying increased activation of TGF $\beta$ signaling in tumor initiating cells (30). Similar to our findings, TGF $\beta$ could induce integrin $\beta 3$ expression when cells form complex spheric organizations.

Involvement between cells and the ECM plays an important role in normal development and differentiation. However, remodeling of the ECM occurs in many pathological states. Changes in the ECM are regulated by a system of proteolytic enzymes that are responsible for the proteolysis of a large quantity of ECM components (31). Previous studies demonstrated that CSCs require these for the maintenance of the extracellular matrix and CSCs express different molecules for detachment from the niche (32-34). In the present study, notably, we determined a significant increase in MMP16 whereas here was a decrease in MMP1 and 14. Astarci et al showed that cell differentiation in the Caco- 2 cell line is accompanied by decreased MMP-16 mRNA and protein expression. This group demonstrated that the forced expression of miR-146a in HT29 colon cancer cells resulted in decreased expression of MMP16 (35). Nevertheless, Inoue et al demonstrated in glioma cells that MMP13 was specifically expressed in tumor sphere-forming cells and that it is related to the invasive potential of CSCs (32). On the other hand, it has been shown in breast cancer cells that cancer progression inhibitors suppress the mRNA expression of some matrix metalloproteinases (MMPs), but stimulate that of others.

Our electron microscopic data support the theory that the predominant cell death mechanism in non-CSCs is autophagy. In spheroids, CSCs lose their villous protrusions and cellular organelles are observed to be clusters on the side of the cytoplasm. During our literature review, we did not find any definite data regarding these electron microscopic results.
In conclusion, the present study demonstrated first that isolated CSCs were found to possess multipotential differentiation capabilities regulated through the upregulation and/or downregulation of their markers, particularly versican, TGF $\beta 1$, Col7A1 and ITG $\beta 3$. The most striking observation is the upregulation of versican that was only determined in CSCs. We assume that one must engage CSCs or more signaling cascades to differentiate and initiate tumor formation. This mechanism occurs with specific intracellular and extracellular signals. It is possible that CSCs themselves may be a source of extracellular signaling molecules. This may be a triggering mechanism for the construction of required extracellular matrix compositions. These molecules that are effective during tumor progression differentiation could be targeted for future therapeutic interventions. Developing new therapeutic strategies that will effectively target this critically important population of cancer cells may be a cornerstone in cancer therapy.

\section{Acknowledgements}

This study was funded by the Ege University Scientific Research Project Fund.

\section{References}

1. Singh SK, Clarke ID, Terasaki M, Bonn VE, Hawkins C, Squire J and Dirks PB: Identification of a cancer stem cell in human brain tumors. Cancer Res 63: 5821-5828, 2003.

2. Yu Z, Pestell TG, Lisanti MP and Pestell RG: Cancer stem cells. Int J Biochem Cell Biol 44: 2144-2151, 2012.

3. Neumüller RA and Knoblich JA: Dividing cellular asymmetry: asymmetric cell division and its implications for stem cells and cancer. Genes Dev 23: 2675-2699, 2009.

4. La Porta CA: Mechanism of drug sensitivity and resistance in melanoma. Curr Cancer Drug Targets 9: 391-397, 2009.

5. Yin G, Alvero AB, Craveiro V, Holmberg JC, Fu HH, Montagna MK, Yang Y, Chefetz-Menaker I, Nuti S, Rossi M, Silasi DA, Rutherford T and Mor G: Constitutive proteasomal degradation of TWIST-1 in epithelial-ovarian cancer stem cells impacts differentiation and metastatic potential. Oncogene 32: 39-49, 2013.

6. Hurt EM, Chan K, Serrat MA, Thomas SB, Veenstra TD and Farrar WL: Identification of vitronectin as an extrinsic inducer of cancer stem cell differentiation and tumor formation. Stem Cells 28: 390-398, 2010.

7. Robertson FM, Ogasawara MA, Ye Z, Chu K, Pickei R, Debeb BG, Woodward WA, Hittelman WN, Cristofanilli M and Barsky SH: Imaging and analysis of 3D tumor spheroids enriched for a cancer stem cell phenotype. J Biomol Screen 15: 820-829, 2010.

8. Vinci M, Gowan S, Boxall F, Patterson L, Zimmermann M, Court W, Lomas C, Mendiola M, Hardisson D and Eccles SA: Advances in establishment and analysis of three-dimensional tumor spheroid-based functional assays for target validation and drug evaluation. BMC Biol 10: 29, 2012.

9. Ayla S, Bilir A, Soner BC, Yilmaz-Dilsiz O, Ergüven M and Oktem G: Notch signaling-related therapeutic strategies with novel drugs in neuroblastoma spheroids. J Pediatr Hematol Oncol 36: 37-44, 2014.

10. Diaz JA and Murillo MF: Phenotype characterization of embryoid body structures generated by a crystal comet effect tail in an intercellular cancer collision scenario. Cancer Manag Res 4: 9-21, 2012.

11. Gaedtke L, Thoenes L, Culmsee C, Mayer B and Wagner E: Proteomic analysis reveals differences in protein expression in spheroid versus monolayer cultures of low-passage colon carcinoma cells. J Proteome Res 6: 4111-4118, 2007.

12. Phillips TM, McBride WH and Pajonk F: The response of $\mathrm{CD} 24^{-/ \text {low }} / \mathrm{CD} 44^{+}$breast cancer-initiating cells to radiation. J Natl Cancer Inst 98: 1777-1785, 2006. 
13. Oktem G, Bilir A, Ayla S, Yavasoglu A, Goksel G, Saydam G and Uysal A: Role of intercellular communications in breast cancer multicellular tumor spheroids after chemotherapy. Oncol Res 16: 225-233, 2006

14. Oktem G, Bilir A, Selvi N, Yurtseven ME, Vatansever S, Ates U, Uysal A and Omay SB: Chemotherapy influences inducible nitric oxide synthase (iNOS) and endothelial nitric oxide synthase (eNOS) activity on 3D breast cancer cell line. Oncol Res 16 195-203, 2006

15. Edwards IJ: Proteoglycans in prostate cancer. Nat Rev Urol 9: 196-206, 2012

16. Ricciardelli C, Sakko AJ, Ween MP, Russell DL and Horsfall DJ: The biological role and regulation of versican levels in cancer. Cancer Metastasis Rev 28: 233-245, 2009.

17. Sakko AJ, Ricciardelli C, Mayne K, Suwiwat S, LeBaron RG, Marshall VR, Tilley WD and Horsfall DJ: Modulation of prostate cancer cell attachment to matrix by versican. Cancer Res 63: 4786-4791, 2003.

18. Ricciardelli C, Russell DL, Ween MP, Mayne K, Suwiwat S, Byers S, Marshall VR, Tilley WD and Horsfall DJ: Formation of hyaluronan- and versican-rich pericellular matrix by prostate cancer cells promotes cell motility. J Biol Chem 282: 10814-10825, 2007.

19. Ween MP, Hummitzsch K, Rodgers RJ, Oehler MK and Ricciardelli C: Versican induces a pro-metastatic ovarian cancer cell behavior which can be inhibited by small hyaluronan oligosaccharides. Clin Exp Metastasis 28: 113-125, 2011.

20. Sakko AJ, Ricciardelli C, Mayne K, Tilley WD, Lebaron RG and Horsfall DJ: Versican accumulation in human prostatic fibroblas cultures is enhanced by prostate cancer cell-derived transforming growth factor $\beta 1$. Cancer Res 61: 926-930, 2001.

21. Cross NA, Chandrasekharan S, Jokonya N, Fowles A, Hamdy FC, Buttle DJ and Eaton CL: The expression and regulation of ADAMTS-1, $-4,-5,-9$, and -15 , and TIMP-3 by TGF $\beta 1$ in prostate cells: relevance to the accumulation of versican. Prostate 63 269-275, 2005.

22. Knaup J, Gruber C, Krammer B, Ziegler V, Bauer J and Verwanger T: TGF $\beta$-signaling in squamous cell carcinoma occurring in recessive dystrophic epidermolysis bullosa. Anal Cell Pathol 34: 339-353, 2011.

23. Ryynänen M, Knowlton RG, Parente MG, Chung LC, Chu ML and Uitto J: Human type VII collagen: genetic linkage of the gene (COL7A1) on chromosome 3 to dominant dystrophic epidermolysis bullosa. Am J Hum Genet 49: 797-803, 1991.
24. Switala-Jelen K, Dabrowska K, Opolski A, Lipinska L, Nowaczyk $M$ and Gorski A: The biological functions of $\beta 3$ integrins. Folia Biol 50: 143-152, 2004.

25. Hood JD and Cheresh DA: Role of integrins in cell invasion and migration. Nat Rev Cancer 2: 91-100, 2002.

26. Hieken TJ, Farolan M, Ronan SG, Shilkaitis A, Wild L and Das Gupta TK: $\beta 3$ integrin expression in melanoma predicts subsequent metastasis. J Surg Res 63: 169-173, 1996.

27. Vacca A, Ria R, Presta M, Ribatti D, Iurlaro M, Merchionne F, Tanghetti $\mathrm{E}$ and Dammacco $\mathrm{F}$ : $\alpha_{\mathrm{v}} \beta_{3}$ integrin engagement modulates cell adhesion, proliferation, and protease secretion in human lymphoid tumor cells. Exp Hematol 29: 993-1003, 2001.

28. Rentala S, Yalavarthy PD and Mangamoori LN: $\alpha 1$ and $\beta 1$ integrins enhance the homing and differentiation of cultured prostate cancer stem cells. Asian J Androl 12: 548-555, 2010.

29. Sagar BM, Rentala S, Gopal PN, Sharma S and Mukhopadhyay A: Fibronectin and laminin enhance engraftibility of cultured hematopoietic stem cells. Biochem Biophys Res Commun 350: 1000-1005, 2006.

30. Lo PK, Kanojia D, Liu X, Singh UP, Berger FG, Wang Q and Chen H: CD49f and CD61 identify Her2/neu-induced mammary tumor-initiating cells that are potentially derived from luminal progenitors and maintained by the integrin-TGF $\beta$ signaling. Oncogene 31: 2614-2626, 2012.

31. Pytliak M, Vargová V and Mechírová V: Matrix metalloproteinases and their role in oncogenesis: a review. Onkologie 35 49-53, 2012

32. Inoue A, Takahashi H, Harada H, Kohno S, Ohue S, Kobayashi K, Yano H, Tanaka J and Ohnishi T: Cancer stem-like cells of glioblastoma characteristically express MMP-13 and display highly invasive activity. Int J Oncol 37: 1121-1131, 2010.

33. Barami K: Relationship of neural stem cells with their vascular niche: implications in the malignant progression of gliomas. J Clin Neurosci 15: 1193-1197, 2008.

34. Calabrese C, Poppleton H, Kocak M, Hogg TL, Fuller C, Hamner B, Oh EY, Gaber MW, Finklestein D, Allen M, Frank A, Bayazitov IT, Zakharenko SS, Gajjar A, Davidoff A and Gilbertson RJ: A perivascular niche for brain tumor stem cells. Cancer Cell 11: 69-82, 2007.

35. Astarci E, Erson-Bensan AE and Banerjee S: Matrix metalloprotease 16 expression is downregulated by microRNA-146a in spontaneously differentiating Caco-2 cells. Dev Growth Differ 54: 216-226, 2012. 